rheuma plus $2018 \cdot 17: 177$

https://doi.org/10.1007/s12688-018-0230-y

Online publiziert: 23. Oktober 2018

(c) Springer-Verlag GmbH Austria, ein Teil von

Springer Nature 2018

\section{Burkhard Leeb}

Leiter des Institutes für Klinische Rheumatologie der Karl Landsteiner Gesellschaft, Hollabrunn, Österreich

\title{
Treat to target - Alles gut?
}

Liebe Leserinnen und Leser!

Sie halten nun die letzte Ausgabe von rheuma plus im Jahr 2018, in dem wir erstmals sechs Mal erschienen sind, in Händen. Wir hoffen auf eine, wie man in Wien sagt, gute Nachrede. In diesem Jahr sind die Therapieoptionen in der Rheumatologie wieder deutlich mehr geworden, orale Präparate haben sich etabliert, neue Indikationen wurden erschlossen, neue Biosimilars sind dazugekommen. Aber ob wir wirklich besser geworden sind? Wir sollten jedenfalls nicht aufhören unsere Strategien und unser Handeln zu hinterfragen.

Treat to target bedeutet ein Ziel zu haben; selbstverständlich hatten Rheumatologen auch vor dessen segenbringender Erfindung eigentlich immer das Ziel, das bestmögliche Behandlungsergebnis für Ihre Patienten zu erreichen. Ziehen wir allerdings die erreichbaren Remissionsraten in Betracht, so erreichen wir unsere Behandlungserfolge höchstwahrscheinlich deshalb, weil uns wesentlich bessere Medikamente zur Verfügung stehen als in früheren Zeiten. Und wer definiert eigentlich das bestmögliche Behandlungsergebnis? Ist es der Betroffene, der alle Auswirkungen der Erkrankung zu tragen hat oder ist es der Arzt, der zwar hoffentlich viel Wissen über die Krankheit hat, aber in der Regel kein Betroffener ist? Das allein zeigt das Dilemma der „shared decision“, die ja in Wirklichkeit keine sein kann. Der Arzt kann nur als erfahrener Ratgeber fungieren, den Weg weisen, die letzte Entscheidung wird immer beim Betroffenen liegen.

Es ist auch zu bedenken, dass unsere therapeutischen Optionen trotz größer werdender Vielfalt nicht unbegrenzt sind. Ein nicht hinterfragendes Einhalten des Prinzips wird irgendwann zu einem Erschöpfen der Möglichkeiten führen, was dann? Treat-totarget entspricht den Erfordernissen der klinischen Forschung, der Welt der klinischen Studien. Will man es in die klinische Routine übertragen, so ist das eine andere Geschichte. Vor allem müssen die Wünsche der Patienten, aber auch die Gegebenheiten der Gesundheitssysteme in Rechnung gestellt werden.

Selbstverständlich haben die Paradigmenwechsel der letzten Jahre verbunden mit den innovativen Medikamenten zu einer massiven Verbesserung der Situation der rheumatologischen Patienten und auch der Rheumatologen geführt, aber alle die Prinzipien tragen in sich das Risiko von $\mathrm{zu}$ viel Diagnostik und auch Überbehandlung, daher ist insgesamt Vorsicht angebracht (Robert Landewe, 2018).

Diesen qualitätssichernden Anstrengungen genügen vor allem die Registerdatenbanken. In dieser Ausgabe werden die Ergebnisse aus BioReg für Spondyloarthritiden und sonstige rheumatologische Erkrankungen präsentiert. Dort wo zukünftiges Leben die wesentliche Rolle bei der Therapiewahl spielt, nämlich bei Schwangerschaft und Stillperiode, kommt Erfahrungsberichten und klinischen Statements mehr als Studienevidenz große Bedeutung zu. Isabell Haase und Rebecca Fischer-Betz aus Düsseldorf liefern ein Update zu diesem Thema.

Misserfolg wurde aus allen klinischen Studien und auch "treat to target“"verbannt. Therapierefraktäre Patienten, die Non-Responder, sind sozusagen selbst schuld, die Parias der Rheumatologie zu sein. Wer sich nicht systemkonform verhält, über den wird nicht gesprochen. Aber behandeln muss sie der Rheumatologe in der Praxis, meist ohne evidenzbasierte Empfehlungen abzielend auf den schwierigen Patienten. Danke an Markus Gaugg, Klagenfurt und St.Veit/ Glan, der sich dieses wirklich schwierigen und auch undankbaren Themas annimmt.

Diagnostische Probleme können immer bei unklaren, untypischen Beschwerdebildern entstehen, häufig bei Paraneoplasien, in dem Report von J. Leipe und. Hendrik Schulze-Koops, München wird diesem wichtigen Thema Raum gegeben.
Wir hoffen, auch mit dieser Ausgabe wieder Ihr Interesse wecken zu können, um dem Ziel von rheuma plus nahe zu kommen, eine fruchtbringende, offene und redliche Diskussion in Gang zu bringen, aus der alle Nutzen ziehen können. Wie immer an dieser Stelle möchten wir Sie herzlich dazu einladen, uns Ihre Meinung zu den Beiträgen dieser Ausgabe, wie auch zu allen rheumatologischen Themen, die Sie für relevant oder für diskussionswert halten, zu schicken. Sie können in jedem Fall auf einen fairen Review mit entsprechendem Feedback vertrauen. Für Kritik, Hinweise, Zustimmung wie auch für jeden anderen Beitrag bedanke ich mich schon im Voraus.

Herzlichst,

Ihr Burkhard Leeb

Korrespondenzadresse
Babogard Leeb
2020 Hollabrunn, Österreich
leeb.rheuma@aon.at
www.leeb-rheuma.at

